\title{
Crystal structure of bis[N,O-1-isoquinolinecarboxylato]copper(II), $\mathrm{C}_{20} \mathrm{H}_{12} \mathrm{CuN}_{2} \mathrm{O}_{4}$
}

\section{O. Pardo, A. Tomas}

Université de Paris V, Faculté des Sciences Pharmaceutiques et Biologiques, Laboratoire de Physique, 4, avenue de l'Observatoire, F-75270 Paris Cedex 06, France

\section{B. Viossat}

Faculté de Médecine et de Pharmacie, Laboratoire de Chimie Générale, 34, rue du Jardin des Plantes, BP199, F-86005 Poitiers Cedex, France

\section{B. Bachet}

Université P. et M. Curie, Laboratoire de Minéralogie - Cristallographie, 4, place Jussieu, F-75230 Paris Cedex 05, France

and D. Nguyen-Huy

Université de Paris XI, Faculté de Pharmacie, Laboratoire de Cristallochimie Bioinorganique, rue Jean-Baptiste Clément, F-92296 Châtenay-Malabry, France

Received July 10, 1998, CSD-No. 409340

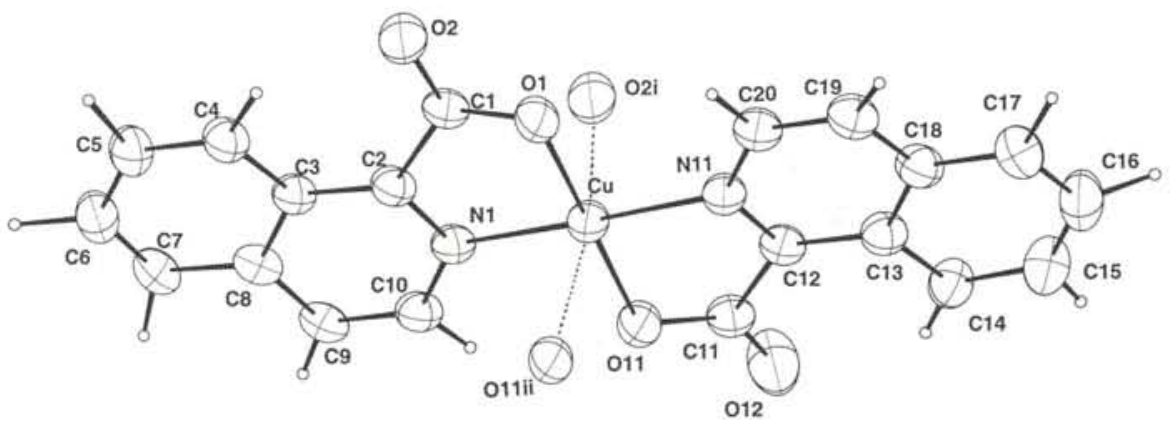

\begin{abstract}
$\mathrm{C}_{20} \mathrm{H}_{12} \mathrm{CuN}_{2} \mathrm{O}_{4}$, monoclinic, $C 12 / c 1$ (No. 15), $a=9.637(3) \AA$, $b=13.453(4) \AA, c=25.163(9) \AA, \beta=97.86(2)^{\circ}, V=3231.6 \AA^{3}$, $Z=8, \rho_{\mathrm{m}}=1.630 \mathrm{~g} \cdot \mathrm{cm}^{-3}, \mathrm{R}_{\mathrm{gt}}(F)=0.064, R_{\mathrm{w}}\left(F^{2}\right)=0.199$, $T=293 \mathrm{~K}$
\end{abstract}

\section{Source of material}

The bis[ $\mathrm{N}, \mathrm{O}$-isoquinoline-1-carboxylato $]$ copper(II) was prepared by adding an aqueous solution of cupric chloride dihydrate to the sodium salt dissolved in water [1]. The resulting purple precipitate was collected by filtration, washed with water and dried overnight. After addition of methanol and concentrated hydrochloric acid (one drop) to dissolve the precipitate, the copper complex crystallized from methanol as purple blocks.

\section{Discussion}

The two isoquinoline-1-carboxylato chelates are bound to $\mathrm{Cu}$ (II) via $\mathrm{N}, \mathrm{O}$ atoms. The $\mathrm{Cu}$ atom exhibits a roughly square planar coordination with the $\mathrm{O}-\mathrm{Cu}-\mathrm{N}$ angles from $82.96(12)^{\circ}$ to $97.04(12)^{\circ}$. The dihedral angle between the two isoquinoline mean planes is $5.29(8)^{\circ}$ and these latter make respectively a dihedral angle of $5.3(1)^{\circ}$ and $9.81(9)^{\circ}$ with the $\mathrm{N}, \mathrm{O}, \mathrm{N}, \mathrm{O}$ mean plane from which copper(II) is displaced by $0.012(2) \AA$.
Table 1. Data collection and handling

$\begin{array}{ll}\text { Crystal: } & \begin{array}{l}\text { violet parallelepiped, } \\ \text { size } 0.125 \times 0.250 \times 0.250 \mathrm{~mm}\end{array} \\ \text { Wavelength: } & \text { Cu } K \alpha \text { radiation }(1.54180 \AA) \\ \mu: & 21.88 \mathrm{~cm}^{-1} \\ \text { Diffractometer, scan mode: } & \text { Philips, } \omega / 2 \theta \\ 2 \theta_{\text {max: }} & 125.96^{\circ} \\ N(h k l)_{\text {measured }}, N(h k l)_{\text {unique: }} & 2681,2615 \\ \text { Criterion for } I_{\text {obs, }} N(h k l)_{\mathrm{gt}}: & I_{\text {obs }}>2 \sigma\left(l_{\text {obs }}\right), 2342 \\ N(\text { param })_{\text {refined: }} & 281 \\ \text { Programs: } & \text { SHELXS-86 [2], SHELXL-93 [3] } \\ & \end{array}$

Table 2. Atomic coordinates and displacement parameters (in $\AA^{2}$ )

\begin{tabular}{llllll}
\hline Atom & Site & $x$ & $y$ & $z$ & $U_{\text {iso }}$ \\
\hline $\mathrm{H}(4)$ & $8 f$ & $0.278(4)$ & $0.748(2)$ & $-0.153(2)$ & $0.075(5)$ \\
$\mathrm{H}(5)$ & $8 f$ & $0.436(5)$ & $0.726(3)$ & $-0.214(2)$ & $0.075(5)$ \\
$\mathrm{H}(6)$ & $8 f$ & $0.594(4) \cdot$ & $0.566(4)$ & $-0.210(2)$ & $0.075(5)$ \\
$\mathrm{H}(7)$ & $8 f$ & $0.552(5)$ & $0.444(2)$ & $-0.131(2)$ & $0.075(5)$ \\
$\mathrm{H}(9)$ & $8 f$ & $0.465(5)$ & $0.404(2)$ & $-0.051(2)$ & $0.075(5)$ \\
$\mathrm{H}(10)$ & $8 f$ & $0.299(6)$ & $0.445(3)$ & $0.009(1)$ & $0.075(5)$ \\
$\mathrm{H}(14)$ & $8 f$ & $-0.072(5)$ & $0.484(2)$ & $0.197(2)$ & $0.075(5)$ \\
$\mathrm{H}(15)$ & $8 f$ & $-0.197(5)$ & $0.525(3)$ & $0.263(1)$ & $0.075(5)$ \\
$\mathrm{H}(16)$ & $8 f$ & $-0.357(5)$ & $0.686(4)$ & $0.256(1)$ & $0.075(5)$ \\
$\mathrm{H}(17)$ & $8 f$ & $-0.383(5)$ & $0.781(3)$ & $0.177(2)$ & $0.075(5)$ \\
$\mathrm{H}(19)$ & $8 f$ & $-0.300(4)$ & $0.800(3)$ & $0.088(2)$ & $0.075(5)$ \\
$\mathrm{H}(20)$ & $8 f$ & $-0.114(6)$ & $0.772(3)$ & $0.033(1)$ & $0.075(5)$ \\
& & & & &
\end{tabular}


Table 3. Atomic coordinates and displacement parameters (in $\AA^{2}$ )

\begin{tabular}{|c|c|c|c|c|c|c|c|c|c|c|}
\hline Atom & Site & $x$ & $y$ & $z$ & $U_{11}$ & $U_{22}$ & $U_{33}$ & $U_{12}$ & $U_{13}$ & $U_{23}$ \\
\hline $\mathrm{Cu}$ & $8 f$ & $0.09083(6)$ & $0.61060(4)$ & $0.01800(2)$ & $0.0519(5)$ & $0.0303(4)$ & $0.0515(5)$ & $0.0049(2)$ & $0.0204(3)$ & $0.0047(2)$ \\
\hline $\mathrm{O}(1)$ & $8 f$ & $0.0731(3)$ & $0.7284(2)$ & $-0.0261(1)$ & $0.057(2)$ & $0.036(2)$ & $0.054(2)$ & $0.011(1)$ & $0.021(1)$ & $0.003(1)$ \\
\hline $\mathrm{O}(2)$ & $8 f$ & $0.1783(4)$ & $0.8082(2)$ & $-0.0861(1)$ & $0.096(2)$ & $0.036(2)$ & $0.070(2)$ & $0.018(2)$ & $0.042(2)$ & $0.017(2)$ \\
\hline $\mathrm{N}(1)$ & $8 f$ & $0.2294(3)$ & $0.5733(2)$ & $-0.0285(1)$ & $0.046(2)$ & $0.029(2)$ & $0.041(2)$ & $0.002(1)$ & $0.008(1)$ & $0.003(1)$ \\
\hline $\mathrm{C}(1)$ & $8 f$ & $0.1575(4)$ & $0.7332(3)$ & $-0.0611(2)$ & $0.052(2)$ & $0.027(2)$ & $0.051(2)$ & $0.007(2)$ & $0.012(2)$ & $0.001(2)$ \\
\hline$C(2)$ & $8 f$ & $0.2430(4)$ & $0.6391(3)$ & $-0.0667(2)$ & $0.042(2)$ & $0.031(2)$ & $0.046(2)$ & $0.003(2)$ & $0.005(2)$ & $-0.001(2)$ \\
\hline$C(3)$ & $8 f$ & $0.3316(4)$ & $0.6233(3)$ & $-0.1056(2)$ & $0.044(2)$ & $0.031(2)$ & $0.042(2)$ & $0.002(1)$ & $0.006(2)$ & $-0.000(1)$ \\
\hline $\mathrm{C}(4)$ & $8 f$ & $0.3417(4)$ & $0.6872(3)$ & $-0.1498(2)$ & $0.058(2)$ & $0.038(2)$ & $0.044(2)$ & $0.006(2)$ & $0.011(2)$ & $0.003(2)$ \\
\hline $\mathrm{C}(5)$ & $8 f$ & $0.4330(5)$ & $0.6668(3)$ & $-0.1848(2)$ & $0.072(3)$ & $0.051(3)$ & $0.043(2)$ & $0.003(2)$ & $0.018(2)$ & $0.001(2)$ \\
\hline$C(6)$ & $8 f$ & $0.5216(5)$ & $0.5826(4)$ & $-0.1780(2)$ & $0.064(3)$ & $0.052(3)$ & $0.051(2)$ & $0.006(2)$ & $0.022(2)$ & $-0.003(2)$ \\
\hline $\mathrm{C}(7)$ & $8 f$ & $0.5153(5)$ & $0.5188(3)$ & $-0.1373(2)$ & $0.054(2)$ & $0.040(2)$ & $0.054(2)$ & $0.007(2)$ & $0.012(2)$ & $-0.007(2)$ \\
\hline $\mathrm{C}(8)$ & $8 f$ & $0.4178(4)$ & $0.5348(3)$ & $-0.1000(2)$ & $0.045(2)$ & $0.033(2)$ & $0.045(2)$ & $0.001(2)$ & $0.005(2)$ & $-0.005(2)$ \\
\hline $\mathrm{C}(9)$ & $8 f$ & $0.4034(4)$ & $0.4684(3)$ & $-0.0581(2)$ & $0.046(2)$ & $0.031(2)$ & $0.047(2)$ & $0.0058(2)$ & $0.004(2)$ & $-0.005(2)$ \\
\hline $\mathrm{C}(10)$ & $8 f$ & $0.3088(4)$ & $0.4881(3)$ & $-0.0241(2)$ & $0.044(2)$ & $0.031(2)$ & $0.046(2)$ & $0.001(2)$ & $0.007(2)$ & $0.001(2)$ \\
\hline$O(11)$ & $8 f$ & $0.1048(3)$ & $0.4917(2)$ & $0.0611(1)$ & $0.057(2)$ & $0.038(2)$ & $0.062(2)$ & $0.011(1)$ & $0.026(1)$ & $0.005(1)$ \\
\hline$O(12)$ & $8 f$ & $0.0728(4)$ & $0.4413(3)$ & $0.1421(1)$ & $0.109(3)$ & $0.063(2)$ & $0.061(2)$ & $0.040(2)$ & $0.034(2)$ & $0.023(2)$ \\
\hline$N(11)$ & $8 f$ & $-0.0417(3)$ & $0.6509(2)$ & $0.0670(1)$ & $0.047(2)$ & $0.031(2)$ & $0.045(2)$ & $0.003(1)$ & $0.010(1)$ & $0.005(1)$ \\
\hline$C(11)$ & $8 f$ & $0.0538(4)$ & $0.4998(3)$ & $0.1058(2)$ & $0.058(2)$ & $0.031(2)$ & $0.052(2)$ & $0.007(2)$ & $0.012(2)$ & $0.006(2)$ \\
\hline $\mathrm{C}(12)$ & $8 f$ & $-0.0423(4)$ & $0.5902(3)$ & $0.1083(2)$ & $0.046(2)$ & $0.031(2)$ & $0.046(2)$ & $0.005(2)$ & $0.008(2)$ & $0.000(2)$ \\
\hline $\mathrm{C}(13)$ & $8 f$ & $-0.1282(4)$ & $0.6077(3)$ & $0.1488(2)$ & $0.040(2)$ & $0.036(2)$ & $0.045(2)$ & $0.002(1)$ & $0.004(2)$ & $0.001(2)$ \\
\hline $\mathrm{C}(14)$ & $8 f$ & $-0.1308(4)$ & $0.5475(3)$ & $0.1946(2)$ & $0.050(2)$ & $0.050(2)$ & $0.046(2)$ & $0.000(2)$ & $0.011(2)$ & $0.005(2)$ \\
\hline$C(15)$ & $8 f$ & $-0.2156(5)$ & $0.5727(4)$ & $0.2317(2)$ & $0.055(3)$ & $0.070(3)$ & $0.051(2)$ & $-0.001(2)$ & $0.017(2)$ & $0.005(2)$ \\
\hline$C(16)$ & $8 f$ & $-0.3019(5)$ & $0.6563(4)$ & $0.2257(2)$ & $0.056(3)$ & $0.074(3)$ & $0.055(2)$ & $0.001(2)$ & $0.023(2)$ & $-0.005(2)$ \\
\hline$C(17)$ & $8 f$ & $-0.3047(5)$ & $0.7155(4)$ & $0.1818(2)$ & $0.055(2)$ & $0.055(3)$ & $0.062(3)$ & $0.007(2)$ & $0.018(2)$ & $-0.005(2)$ \\
\hline $\mathrm{C}(18)$ & $8 f$ & $-0.2168(4)$ & $0.6943(3)$ & $0.1422(2)$ & $0.047(2)$ & $0.038(2)$ & $0.049(2)$ & $0.003(2)$ & $0.007(2)$ & $-0.004(2)$ \\
\hline$C(19)$ & $8 f$ & $-0.2139(4)$ & $0.7540(3)$ & $0.0971(2)$ & $0.053(2)$ & $0.037(2)$ & $0.055(2)$ & $0.007(2)$ & $0.008(2)$ & $0.001(2)$ \\
\hline $\mathrm{C}(20)$ & $8 f$ & $-0.1253(4)$ & $0.7332(3)$ & $0.0614(2)$ & $0.055(2)$ & $0.033(2)$ & $0.053(2)$ & $0.005(2)$ & $0.013(2)$ & $0.003(2)$ \\
\hline
\end{tabular}

\section{References}

1. Sorenson, J. R. J.: Copper Chelates as Possible Active Forms of the Antiarthritic Agents. J. Med. Chem. 19 (1976) 135-148.
2. Sheldrick, G. M.: SHELXS-86. Program for solution of crystal structures. University of Göttingen Germany 1985.

3. Sheldrick, G. M.: SHELXL-93. Program for refining crystal structures. University of Göttingen Germany 1993. 